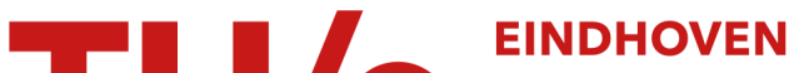 \\ UNIVERSITY OF \\ TECHNOLOGY
}

\section{Spin-up in a rectangular container with an internal cylindrical obstacle}

Citation for published version (APA):

Suh, Y. K., \& Heijst, van, G. J. F. (2000). Spin-up in a rectangular container with an internal cylindrical obstacle. Physics of Fluids, 12(8), 1986-1996. https://doi.org/10.1063/1.870446

DOI:

10.1063/1.870446

Document status and date:

Published: 01/01/2000

Document Version:

Publisher's PDF, also known as Version of Record (includes final page, issue and volume numbers)

Please check the document version of this publication:

-A submitted manuscript is the version of the article upon submission and before peer-review. There can be important differences between the submitted version and the official published version of record. People interested in the research are advised to contact the author for the final version of the publication, or visit the $\mathrm{DOI}$ to the publisher's website.

- The final author version and the galley proof are versions of the publication after peer review.

- The final published version features the final layout of the paper including the volume, issue and page numbers.

Link to publication

\section{General rights}

Copyright and moral rights for the publications made accessible in the public portal are retained by the authors and/or other copyright owners and it is a condition of accessing publications that users recognise and abide by the legal requirements associated with these rights.

- Users may download and print one copy of any publication from the public portal for the purpose of private study or research.

- You may not further distribute the material or use it for any profit-making activity or commercial gain

- You may freely distribute the URL identifying the publication in the public portal.

If the publication is distributed under the terms of Article $25 \mathrm{fa}$ of the Dutch Copyright Act, indicated by the "Taverne" license above, please follow below link for the End User Agreement:

www.tue.nl/taverne

Take down policy

If you believe that this document breaches copyright please contact us at:

openaccess@tue.nl

providing details and we will investigate your claim. 


\title{
Spin-up in a rectangular container with an internal cylindrical obstacle
}

Y. K. Suh

Department of Mechanical Engineering, Dong-A University, Pusan 604-714, Korea

G. J. F. van Heijst

J.M. Burgers Centre for Fluid Mechanics, Department of Technical Physics, Eindhoven University of Technology, Eindhoven, The Netherlands

(Received 6 January 1999; accepted 5 April 2000)

\begin{abstract}
This paper describes a study of the spin-up of a free-surface fluid in a rectangular container in which an internal cylindrical obstacle is mounted. Laboratory experiments have been carried out for a variety of obstacle positions. It was found that the flow evolution during the adjustment process leading to the final state of solid-body rotation is crucially dependent on the obstacle position. As found in previous studies, in the absence of any obstacle the relative flow becomes organized in a domain-filling regular cellular pattern, upon which it decays according to the well-known spin-up mechanism provided by the Ekman layer at the tank bottom. Since the obstacle acts both as a barrier and as a source of viscously produced wall vorticity, the formation of the cell pattern is in most cases drastically influenced (either impeded or promoted) by the solid obstacle. Theoretical predictions of the flow pattern in the starting stage agree very well with the laboratory observations.

(C) 2000 American Institute of Physics. [S1070-6631(00)01707-4]
\end{abstract}

\section{INTRODUCTION}

The spin-up of a fluid in a container, i.e., the adjustment of the contained fluid to any change in the rotation speed $\Omega$ of the system, is an intriguing process, in particular when the container is nonaxisymmetric. Usually "spin-up" refers to the adjustment process owing to an increase in the rotation speed $(\Delta \Omega>0)$, whereas "spin-down" refers to the case of a decrease in the rotation rate $(\Delta \Omega<0)$. When $\Delta \Omega \ll \Omega$, the advective terms in the equation of motion are negligible, and the spin-up process is called "linear." Greenspan and Howard ${ }^{1}$ performed a pioneering study, and constructed an analytical solution for the spin-up of fluid between two parallel, infinitely large plates rotating about a common axis. The Ekman boundary layers present at the horizontal flow boundaries play a crucial role in the spin-up process: The radially outward motion in the Ekman layers induces a weak secondary circulation (the primary circulation being the rotational motion of the fluid), in the interior, consisting of a weak radial contraction of the fluid between the layers. Conservation of angular momentum causes the radially contracting fluid to acquire a larger angular velocity, until the fluid rotates with the new rotation rate. It was shown that this stage is reached on a time scale $\tau=H /(v \Omega)^{1 / 2}$, with $2 H$ the distance between the plates, and $v$ the kinematic viscosity.

The nonlinear spin-up process in a circular tank is much more complicated, since an analytical description of the Ekman layer flow is lacking when the nonlinear terms are nonzero. Aspects of nonlinear spin-up in an axisymmetric container have been studied both analytically, numerically, and experimentally (see, e.g., Refs. 2-7).

The adjustment process becomes even more complicated when the fluid is confined in a nonaxisymmetric tank, even for the linear case $\Delta \Omega \ll \Omega$. In a number of studies ${ }^{8-15}$ the effects of nonaxisymmetry on the spin-up have been inves- tigated for a variety of container shapes, ranging from semicircular, rectangular, circular with radial barrier and annulus with radial barrier. In general, the flow is no longer axisymmetric, and a very complicated flow evolution is observed. In the context of the present paper it is instructive to consider the case of a rectangular tank, as first studied by Van Heijst, Davies, and Davis ${ }^{9}$ (henceforth referred to as vHDD). It was found experimentally that during the spin-up of a freesurface fluid a number of stages can be distinguished: (i) the initial stage, characterized by a uniform relative vorticity (with value $-2 \Delta \Omega$ ) and a thin shear layer at the lateral boundaries (and, of course, an Ekman layer at the tank bottom); (ii) the separation stage, characterized by flow detachment from the lateral tank walls; (iii) the organization stage, during which the quasi-two-dimensional motion in the interior of the domain is observed to become organized in a linear array of counter-rotating cells that fill the rectangular domain completely; and (iv) the decay stage, characterized by the gradual decay of the relative motion in the cells due to the spin-up-spin-down mechanism provided by the Ekman layer at the tank bottom.

The self-organization of the flow into a cellular pattern is an intriguing feature, which is attributed to the quasi-twodimensional (2D) character of the flow throughout the adjustment process. It was found recently (see, e.g., Ref. 16) that the self-organization of quasi-2D flow in a rectangular container into a cell pattern is a rather autonomous process, independent of the details of the initial (global) forcing. According to the inverse energy cascade, which characterizes (quasi-) 2D flows, vortices have the tendency to grow in size. The width of the rectangular domain, however, puts a physical restriction on the size the vortices may reach. Highresolution spectral flow simulations have revealed the crucial role of the lateral no-slip walls in the establishment of the 
organized flow pattern: Viscously produced wall vorticity filaments are advected by the larger-scale vortices away from the walls, where they affect the interior flow evolution.

An obvious question that arises concerns the robustness of the cellular pattern that is formed during the spin-up in the rectangular geometry. It was found both experimentally and numerically ${ }^{17-19}$ that the presence of bottom topography may seriously affect the formation of cellular patterns. For example, a steeply sloping bottom may prevent the forming of any stationary flow pattern. ${ }^{17}$ On the other hand, a suitably arranged step-like bottom topography may promote the formation of a cellular pattern. ${ }^{18}$

It is anticipated that an internal solid obstacle in the flow domain may play a similar role: It may either prevent or promote the establishment of a cell pattern. In the former case, the relative flow is expected to reveal unsteadiness throughout the course of the spin-up to solid-body rotation, while in the latter case, the obstacle may quickly drive the flow into an organized state of a stable array of cells. Once the role of such an internal obstacle is better understood, it may enable one to "control" the flow during spin-up in rotating containers in order to enhance or decrease its mixing efficiency.

The present paper addresses the effect of a solid obstacle on the flow evolution during spin-up for the special case of a rectangular container. The attention is restricted to a cylindrical obstacle with a circular cross section, which is placed vertically-i.e., axially aligned-in the fluid. It is to be expected that a solid obstacle may play a twofold role in the spin-up process: (i) It acts as a solid barrier, thus possibly hampering (or promoting) the formation of a cellular pattern, and (ii) its viscously produced wall vorticity-advected in a filamentary fashion by the larger eddies-may locally affect the formation of cells. It is obvious that, apart from its relative size, the obstacle's position in the rectangular domain will be a crucial factor. The aim of the present paper is to examine experimentally how the spin-up process, and in particular the self-organization of the relative flow, is influenced by the presence of the solid cylinder. Once the effect of the obstacle on the flow evolution has been understood, the flow may thus be controlled in order to enhance or diminish its mixing efficiency. This latter effect may be of relevance to industrial situations.

The plan of the paper is as follows. The experimental arrangement is described in Sec. II. In Sec. III a theoretical analysis of the flow pattern in the starting stage is presented. Experimental results, both for the starting pattern and for the subsequent evolution, are presented in Sec. IV. Some flow stability considerations are given in Sec. V, and a summary of the results is finally presented in Sec. VI.

\section{EXPERIMENT}

The experimental setup is sketched in Fig. 1. The turntable can be rotated in the counter-clockwise direction with a rotational speed $\Omega$ that is adjustable in the range from 0 to $\sim 2 \mathrm{rad} / \mathrm{s}$. A rectangular tank of length $199 \mathrm{~cm}$ and width 40 $\mathrm{cm}$ was mounted on the table, its center being adjusted to coincide with the table's rotational axis. The tank was filled

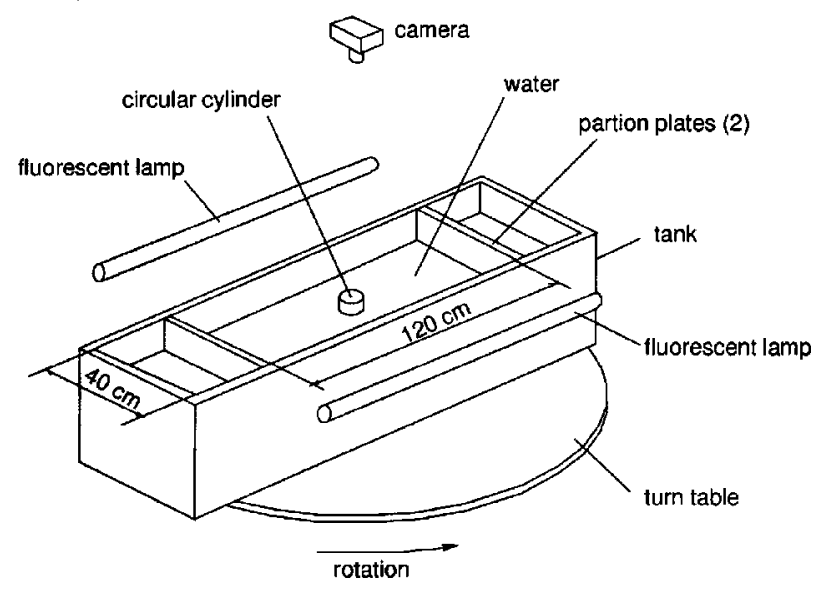

FIG. 1. The experimental setup.

with tap water to a depth of $20 \mathrm{~cm}$, and two partition plates were inserted so that the length $(L)$ of the flow domain became $L=120 \mathrm{~cm}$, while the width $(B)$ was kept at $B$ $=40 \mathrm{~cm}$; that is, we consider a rectangular domain with aspect ratio $a=L / B=3$. A circular cylinder of diameter $12 \mathrm{~cm}$ and height slightly larger than the water depth was then inserted into the tank at some desired location. In order to obtain a better contrast in the flow visualizations, the fluid was colored with a dark blue dye. Information about the evolving flow structure was obtained from small paper particles of 2 to $3 \mathrm{~mm}$ in diameter that were sprinkled all over the water surface. Two fluorescent lamps were mounted along the longer sides of the container, by which the tracer particles were illuminated. A remote-control photocamera was mounted in a co-rotating frame at some distance above the water surface.

The experimental procedure is simple. From a quiescent state the table is suddenly set into rotation: Within one revolution it reaches a final angular speed $\Omega=0.47 \mathrm{rad} / \mathrm{s}$, which is thereafter kept at this value. The corresponding rotation period measures $T=13.5 \mathrm{~s}$. Trajectories of the tracer particles are recorded by the camera at chosen intervals. The exposure time was adjusted from $2.5 \mathrm{~s}$ until $\sim 3$ min from starting ( $t$ $<14 T)$, $4.5 \mathrm{~s}$ after $4.5 \mathrm{~min}(t>21 T)$ and $3.5 \mathrm{~s}$ for the intermediate interval $(14 T<t<21 T)$.

In this section the position of the circular cylinder is specified by two horizontal coordinates $X$ and $Y$ measured along the long and short sides of the tank, respectively, see Fig. 2; in Sec. IV, we use dimensionless coordinates for the cylinder's position. The problem configuration is antisymmetric; for instance, inserting the cylinder at $Y=13 \mathrm{~cm}, X$ $=40 \mathrm{~cm}$ is equivalent to inserting it at the antisymmetric point, that is, at $Y=(B-13)=27 \mathrm{~cm}, X=(L-40)=80 \mathrm{~cm}$. We choose two vertical positions for the visualization study; $Y=20$ and $13 \mathrm{~cm}$, the former corresponding to the case when the cylinder is on the centerline " $\mathrm{CH}$ " (Fig. 2). At $Y$ $=20 \mathrm{~cm}, X$ is varied as $60,50,40,35,30,20$, and $14 \mathrm{~cm}$, while at $Y=13 \mathrm{~cm}, X$ is varied as $100,80,60,40$, and $20 \mathrm{~cm}$.

The Reynolds number is here defined as $\operatorname{Re}=\Omega B^{2} / v$, where $v$ is kinematic viscosity of the liquid. For the experiments reported here, it takes a value of approx. $R e=75000$. 


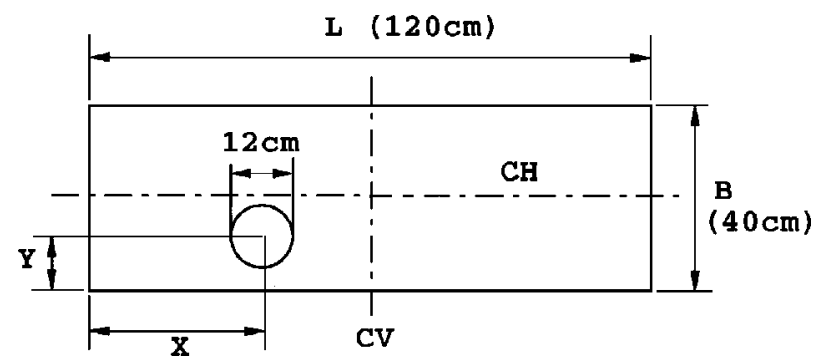

FIG. 2. Geometry of the tank and the relative position $(X, Y)$ of the circular cylinder. Here " $\mathrm{CH}$ " and " $\mathrm{CV}$ " denote the long and short axes of the tank, respectively.

\section{ANALYSIS OF THE STARTING FLOW}

In this section, we will derive the starting flow solution. The equation for the relative vorticity $\boldsymbol{\omega}=\nabla \times \mathbf{u}$, where $\mathbf{u}$ is the velocity field of the relative flow motion within the tank, can be written as (see, e.g., Pedlosky ${ }^{20}$ )

$$
\frac{d \boldsymbol{\omega}}{d t}=\boldsymbol{\omega}_{a} \cdot \nabla \mathbf{u}-2 \frac{d \boldsymbol{\Omega}}{d t},
$$

where $\boldsymbol{\omega}_{a}=\boldsymbol{\omega}+2 \boldsymbol{\Omega}$ is the absolute vorticity, and $\boldsymbol{\Omega}$, the angular velocity of the tank, is considered to be a function of time, the final value being $\Omega$. In this equation the viscous terms are neglected in view of the fact that at the beginning of the spin-up process the viscous effect is confined to the thin boundary layers adjacent to the walls. The last term is added here because this is the only term that generates the relative motion of the inviscid flow; in fact, an impulsive start of rotation yields an infinite value of $d \boldsymbol{\Omega} / d t$ for an infinitesimal duration of time. Since $\boldsymbol{\Omega}$ is free of the spatial coordinates, we can write (1) as

$$
\frac{d \boldsymbol{\omega}_{a}}{d t}=\boldsymbol{\omega}_{a} \cdot \nabla \mathbf{u}
$$

This admits a solution $\boldsymbol{\omega}_{a}=0$, or

$$
\boldsymbol{\omega}=-2 \boldsymbol{\Omega},
$$

indicating that the starting flow has a uniform vorticity equal to $-2 \boldsymbol{\Omega}$. We should note that in deriving this simple result the fluid is assumed to be inviscid. Furthermore, it is assumed that the starting flow is $2 \mathrm{D}$, as is evident from the experimental results. Scaling the spatial coordinates by $B$, velocities by $\Omega B$, and the stream function by $\Omega B^{2}$ results in the following nondimensional equation:

$$
\frac{\partial^{2} \psi}{\partial x^{2}}+\frac{\partial^{2} \psi}{\partial y^{2}}=2
$$

where $\psi$ is now the nondimensional stream function, and $(x, y)$ are the dimensionless coordinates. The boundary conditions are

$$
\begin{aligned}
& \psi=0 \quad \text { at } x=0, a \text { and } y=0,1, \\
& \psi=\text { constant at }\left(x-x_{c}\right)^{2}+\left(y-y_{c}\right)^{2}=\rho^{2},
\end{aligned}
$$

where $a$ is aspect ratio of the tank,

$$
\left(x_{c}, y_{c}\right)=(X / B, Y / B)
$$

represents the dimensionless center position of the circular cylinder, and $\rho$ is its dimensionless radius.

The analysis of this problem starts by considering a simple basis flow. In the limit $a \rightarrow \infty$ and with the circle being absent, the flow is characterized by a uniform shear flow, i.e., $\psi=y(y-1)$. Inserting a circle to this flow field then results, by applying Milne-Thomson's second circle theorem, ${ }^{21}$ in

$$
\psi=\left(2 y_{c}-1\right) \bar{y}\left[1-\frac{\rho^{2}}{\bar{x}^{2}+\bar{y}^{2}}\right]+\left[\bar{y}^{2}+\frac{\rho^{4}\left(\bar{x}^{2}-\bar{y}^{2}\right)}{2\left(\bar{x}^{2}+\bar{y}^{2}\right)^{2}}\right],
$$

where $\bar{x}=x-x_{c}$ and $\bar{y}=y-y_{c}$. Note that the velocity tends to zero at the centerline $y=1 / 2$ for $|x| \rightarrow \infty$. Also note that this flow field satisfies the Eq. (4) and the boundary condition (5b). Thus, to satisfy the remaining boundary condition (5a), a potential flow contribution $\psi_{p}$ must be added.

To obtain $\psi_{p}$, we let

$$
\psi_{p}=\operatorname{Imag}\left[f(z)+\bar{f}\left(\rho^{2} /\left(z-\bar{z}_{c}\right)+z_{c}\right)\right],
$$

where $z=x+i y$ is a complex coordinate, and

$$
\begin{aligned}
f(z)= & A z^{2}+B z+i C z+D \\
& +\sum_{n=1}^{\infty}\left(E_{n} \sinh n \pi z+F_{n} \cosh n \pi z\right. \\
& \left.+G_{n} \sinh i n \pi z / a-H_{n} \cosh i n \pi z / a\right),
\end{aligned}
$$

where $A, B, \ldots, H$ denote real coefficients. The first four terms are added to let $\psi$ become zero in the four corners, so that the following series becomes simpler. In terms of real coordinates, we can compactly write $\psi$ as

$$
\begin{aligned}
\psi= & \psi_{\omega}+2 A[x y-g(x, y) h(x, y)]+B[y-h(x, y)] \\
& +C[x-g(x, y)]+2 D+\sum_{n=1}^{\infty}\left[\left(E_{n} \sinh n \pi(a-x)\right.\right. \\
& \left.+F_{n} \sinh n \pi x\right) \frac{\sin n \pi y}{\sinh n \pi a}+\left(G_{n} \sinh n \pi(1-y) / a\right. \\
& \left.\left.+H_{n} \sinh n \pi y / a\right) \frac{\sin n \pi x / a}{\sinh n \pi / a}\right]+\hat{\psi},
\end{aligned}
$$

where

$$
\begin{aligned}
\psi_{\omega}= & \bar{y}^{2}+\frac{\rho^{4}\left(\bar{x}^{2}-\bar{y}^{2}\right)}{2 r^{4}}, \\
\hat{\psi}= & -\sum_{n=1}^{\infty}\left[\left(E_{n} \sinh n \pi(a-g(x, y))\right.\right. \\
& \left.+F_{n} \sinh n \pi g(x, y)\right) \frac{\sin n \pi h(x, y)}{\sinh n \pi a} \\
& +\left(G_{n} \sinh n \pi(1-h(x, y)) / a\right. \\
& \left.\left.+H_{n} \sinh n \pi h(x, y) / a\right) \frac{\sin n \pi g(x, y) / a}{\sinh n \pi / a}\right],
\end{aligned}
$$


(a) $x_{c}=1.5, y_{c}=0.5$

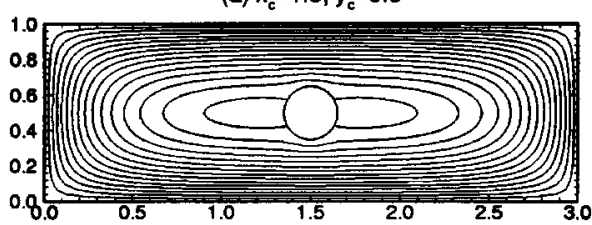

(b) $x_{c}=1.0, y_{c}=0.5$

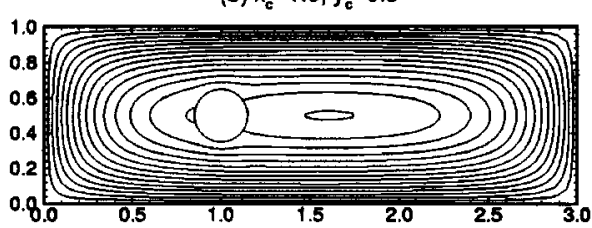

(c) $x_{c}=0.5, y_{c}=0.5$

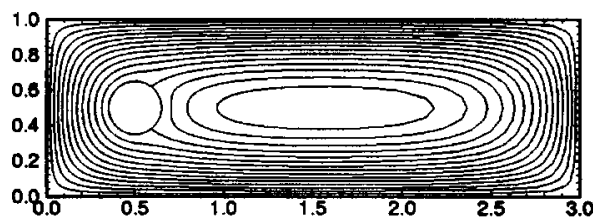

$$
\begin{aligned}
& g(x, y)=\frac{\rho^{2}}{r^{2}} \bar{x}+x_{c}, \quad h(x, y)=\frac{\rho^{2}}{r^{2}} \bar{y}+y_{c}, \\
& r^{2}=\bar{x}^{2}+\bar{y}^{2} .
\end{aligned}
$$

In fact, the first term of Eq. (7) has been absorbed in the term with the constant $B$ in Eq. (10). Evaluation on the circle gives $\psi=\psi_{\text {cir }}=\rho^{2} / 2+2 D=$ constant.

The coefficients in Eq. (10) must be determined such that the impermeability condition at four sides of the rectangle, (5a), is satisfied. We used a numerical method to obtain these coefficients. Although the algebraic equations for these unknowns are basically linear, we found that an iteration strategy is most convenient with a fast convergence property. One iteration process is mainly composed of two steps. In the first, the four unknowns $A, B, C$, and $D$ are solved simultaneously with all the others provided with old values. In the second, the coefficients $E_{n}, F_{n}, G_{n}$, and $H_{n}$ shown explicitly in (10) are solved simultaneously with all the others [including $E_{n}, F_{n}, G_{n}$, and $H_{n}$ in the expression for $\hat{\psi},(11 b)]$ assigned with old values. The computational procedure is summarized as follows:

(1) Truncate the series up to $n=N$;

(2) Set all the coefficients zero;

(3) Obtain $A, B, C$, and $D$ by setting $\psi=0$ at the four corners, with $E_{n}, F_{n}, G_{n}$, and $H_{n}$ in $\hat{\psi}$ assumed to be given;

(4) By setting $\psi=0$ along four sides of the rectangle the coefficients $E_{n}, F_{n}, G_{n}$, and $H_{n}$ can be obtained through Fourier series conversion, again with $E_{n}, F_{n}$, $G_{n}$, and $H_{n}$ in $\hat{\psi}$ assumed to be given;

(5) Repeat the steps (4) and (5) until convergence is obtained. (d) $x_{c}=1,5, y_{c}=0.325$

FIG. 3. Theoretical streamlines of starting flows according to (10) for $a$ $=3$ and $\rho=0.15$. The increment $\Delta \psi$ in these plots is in the range $0.00766-$ 0.00814 and adjusted such that one streamline intersects the cylinder surface.
The streamline plots of the starting flow in (6) of the experimental cases are shown in Fig. 3. When the cylinder is placed on the centerline " $\mathrm{CH}$ ', i.e., $y_{c}=0.5$ (see Fig. 2), the flow is symmetric with respect to the long axis $(\mathrm{CH})$ of the tank. For $x_{c}=1.5$ two small eddies of equal size are situated on either side of the cylinder [Fig. 3(a)], while their sizes become disparate as the cylinder approaches one of the shorter walls [see Fig. 3(b)]. When the cylinder is close enough to either of the end walls, the smallest eddy disappears [Fig. 3(c)]. When the cylinder is located off the long axis of the tank, the eddies are detached from the cylinder [Figs. 3(d) and 3(e)].

When the cylinder size is large compared to the width of the container and the rectangle is close to a square, the basis flow (11a) is no longer optimal, and the convergence becomes slow. In this case, it is better to use

$$
\psi_{\omega}=\frac{1}{2}\left(\bar{x}^{2}+\bar{y}^{2}\right)
$$

which corresponds to a solid-body rotation around the circle. It turns out that using this basis function makes the convergence much faster than the previous one. We obtained solutions for the square domain, $a=1$, and it was found that no eddies are present unless $\rho$ is greater than 0.48. Streamline plots for the case $a=1$ and $\rho$ very close to 0.5 are shown in Fig. 4; these plots show only one quadrant of the square, since the patterns in the other quadrants are identical. One can see that the eddy is very small at $\rho=0.49$, but it becomes large (while $\psi_{\text {cir }}$, which is $\psi$ evaluated on the circle, tends to zero), as $\rho$ approaches 0.5 . This implies that the flow pattern switches from the type "flow-around-cylinder' passing through the corner regions to a recirculatory flow in each corner region.

Thus, it may be interesting to investigate how the passing-flow rate $\psi_{\text {cir }}$ depends on the gap spacing $\Delta \rho$ 


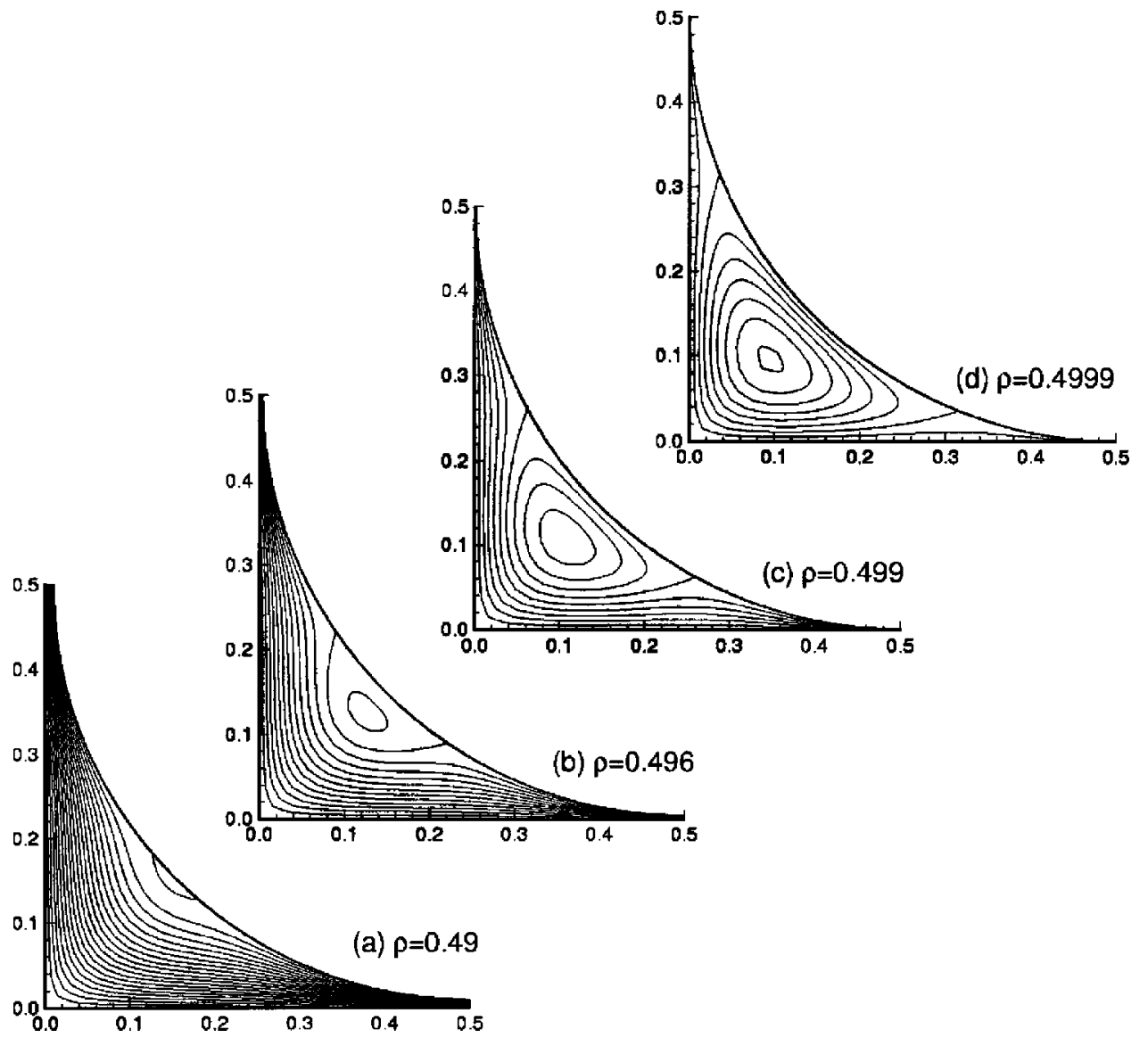

FIG. 4. Starting flows in a quadrant of a square, i.e., $a=1$ with a circular obstacle that is almost touching the sides of the square. The increment $\Delta \psi$ in these plots is in the range 0.000354 0.000402 .

$=0.5-\rho$. If the rectangle were replaced by a circle with radius 0.5 , so that the gap space is uniform along the boundary, then the flow rate between these two boundaries would be

$$
\psi_{\mathrm{cir}}=-\frac{\Delta \rho}{2}(1-\Delta \rho) \text {. }
$$

For $\Delta \rho$ small, this means

$$
\left|\psi_{\text {cir }}\right| \sim \Delta \rho .
$$

A logarithmic plot of the dependence of $\left|\psi_{\text {cir }}\right|$ on $\Delta \rho$ calculated from the full analytical solution is presented in Fig. 5 (the circles indicate the calculated values). From the graph it is inferred that

$$
\left|\psi_{\mathrm{cir}}\right| \sim \sqrt{\Delta \rho} .
$$

The line without symbols in the figure corresponds to the approximating solution (13). The graph reveals that in the limit $\Delta \rho \rightarrow 0$, the passing-flow rate is higher than in the case where the rectangle is replaced by a circle of radius 0.5 . Interestingly, the situation is reversed for $\rho$ less than 0.465 , which is the $\rho$-value at which the two lines in Fig. 5 intersect.

It should be stressed that the analysis of the starting flow, as presented in this section, is only valid for the very first stage of the spin-up process, in which the boundary layers at the lateral boundaries of the flow domain are still very thin. Of course, the boundary layers will quickly grow and flow separation will set in-thus invalidating the analytical approach taken here.

\section{EXPERIMENTAL RESULTS}

Figure 6 shows streak photographs of the experimental starting flow patterns as observed in six different configurations (corresponding with those in Fig. 3). The characteristics of the observed patterns are in good agreement with the corresponding theoretical streamline patterns (cf. Fig. 3). For the cylinder positions $y_{c}=0.325$, i.e., the sets (d)-(f), slight

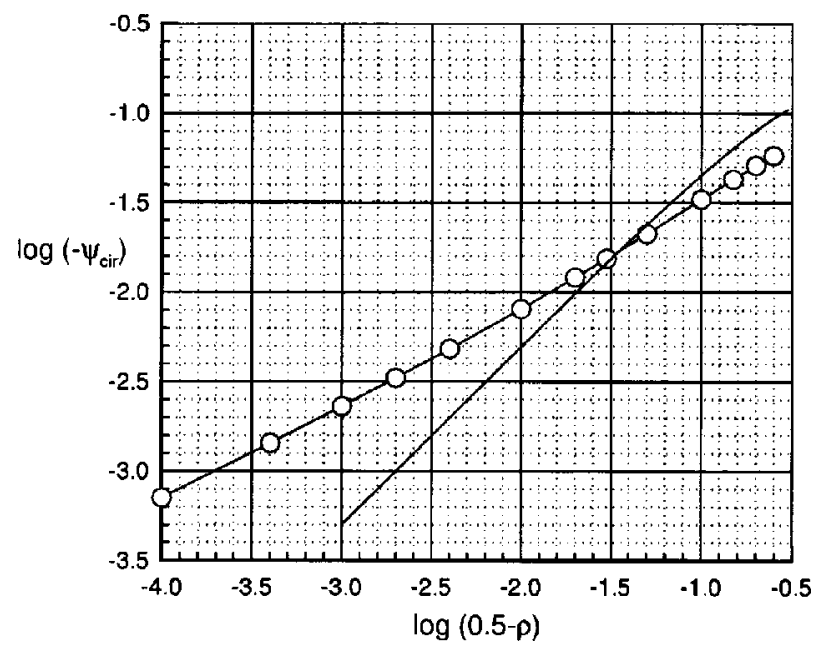

FIG. 5. The passing-flow rate vs the gap spacing in the starting flow at $a$ $=1$ and $\rho=0.15$ calculated from (10) (line with symbols). The line without symbols corresponds to the case when the square domain is replaced by a circle of radius 0.5 for which the approximating solution (13) is derived. 
(a)

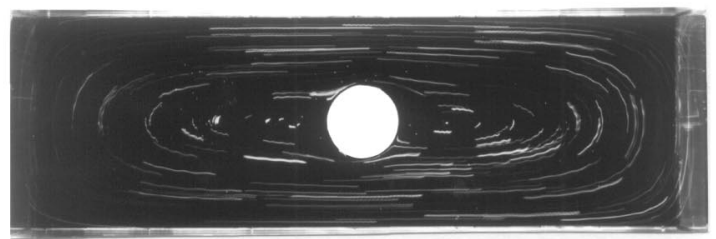

(b)

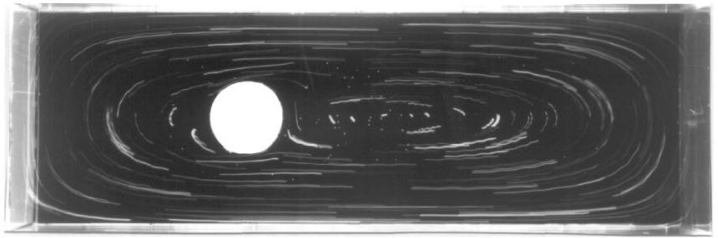

(c)

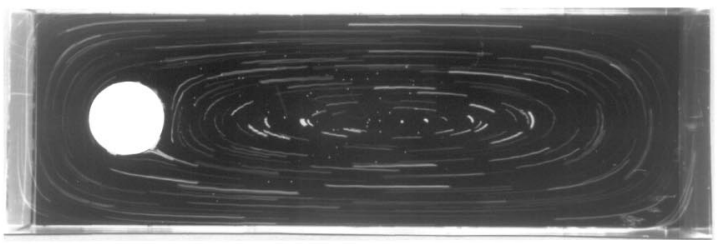

(d)

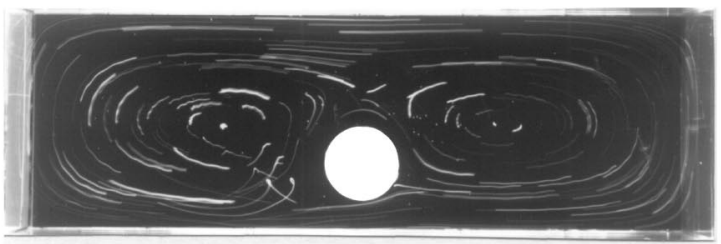

(e)

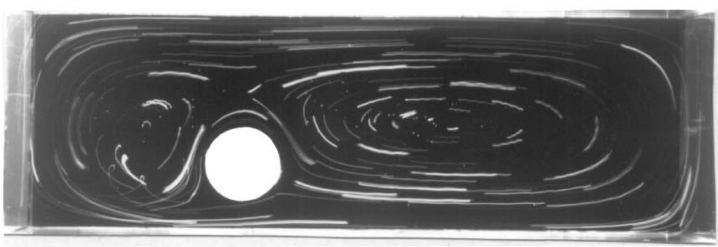

(f)

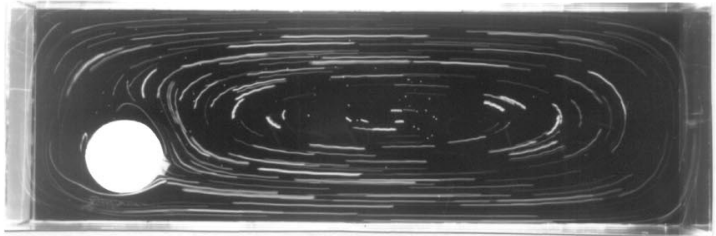

FIG. 6. Streak photographs of experimental starting flow patterns in a rectangular tank $(L=3 B=120 \mathrm{~cm})$ with the inner cylinder (diameter $12 \mathrm{~cm}$ ) positioned at $y_{c}=0.5$ and $x_{c}=1.5$ (a), $1.0(\mathrm{~b}), 0.5$ (c) and at $y_{c}=0.325$ and $x_{c}=1.5(\mathrm{~d}), 1.0(\mathrm{e})$ and $0.5(\mathrm{f})$. Rotation rate $\Omega=0.47 \mathrm{rad} / \mathrm{s}$. The exposure time was $2.5 \mathrm{~s}$ in all cases.

discrepancies are observed on the left-hand side of the internal cylinder: In the experiment the visualized cells are somewhat larger than those predicted theoretically. The larger cell size is attributed to the feeding of negative vorticity from the lower side of the cylindrical obstacle, associated with boundary-layer separation, during the initial stage of the experiment.

The subsequent evolution of the flow during spin-up in the symmetric configuration $\left(x_{c}, y_{c}\right)=(1.5,0.5)$, i.e., with the cylindrical obstacle positioned in the center of the tank, is illustrated by the streak photographs shown in Fig. 7. The pictures reveal that-soon after the initial starting stage shown in Fig. 6(a) - two larger cyclonic cells are formed in the corners downstream of the longer side walls, see Fig. (a)

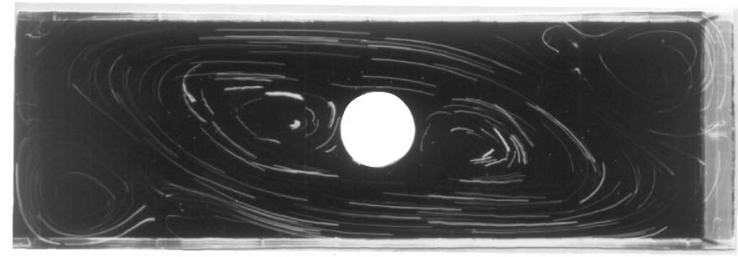

(b)

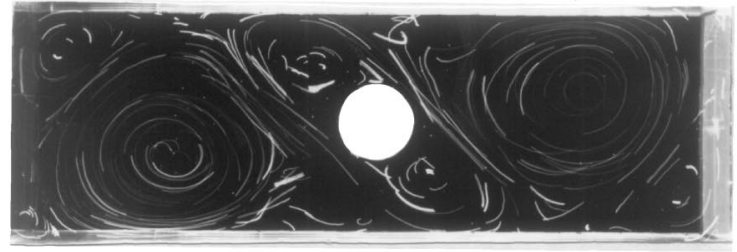

(c)

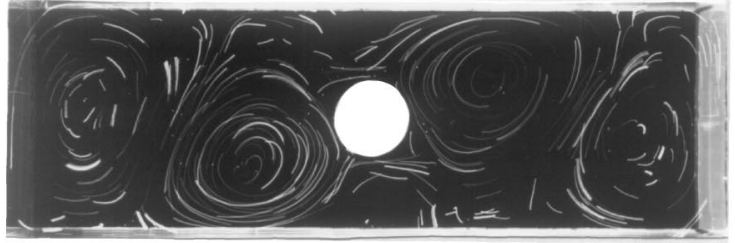

(d)

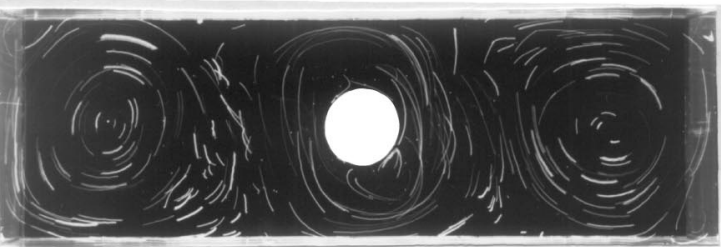

(e)

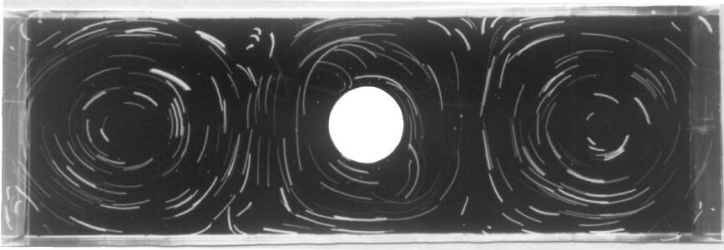

(f)

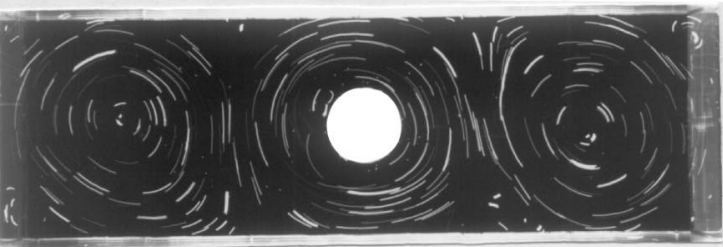

FIG. 7. Sequence of streak photographs illustrating the flow evolution in the case $\left(x_{c}, y_{c}\right)=(1.5,0.5)$ for $t=15 \mathrm{~s}(\mathrm{a}), 30 \mathrm{~s}(\mathrm{~b}), 45 \mathrm{~s}(\mathrm{c}), 55 \mathrm{~s}(\mathrm{~d}), 1 \mathrm{~min} 20$ $\mathrm{s}(\mathrm{e}), 2 \mathrm{~min}(\mathrm{f})$. The exposure time is $2.5 \mathrm{~s}$.

$7(\mathrm{a})$, similar to what one observes during spin-up in absence of an internal obstacle (see vHDD). For convenience the left and right corner cells are referred to as $L^{+}$and $R^{+}$, respectively, with the plus-sign indicating the cyclonic circulation of these cells. Next, the cells $L^{+}$and $R^{+}$are seen to grow quickly [Fig. 7(b)], causing the central cell $\left(C^{-}\right)$to split. While growing, the cells $L^{+}$and $R^{+}$move to the center of the tank [Fig. 7(c)] where they finally merge [Fig. 7(d)] into a single cell centered around the cylindrical obstacle. At this stage, the flow has become organized into a quasi-steady pattern of three equally sized cells: The central cyclonic cell is flanked by two anticyclonic cells. These anticyclonic cells originate from the splitting of the original central cell [see Fig. 7(b)], and were subsequently fed by viscously produced 
negative wall vorticity associated with the cyclonic cells $L^{+}$ and $R^{+}$. Once the three-cell pattern has been established no topological changes are observed, and the flow gradually slows down due to the spin-up-spin-down mechanism provided by the Ekman layer at the tank bottom. In this case the overall evolution of the flow pattern is very similar to that encountered in the absence of the cylindrical obstacle (see vHDD).

Experiments were also carried out for the cylinder positioned slightly off-center, at $\left(x_{c}, y_{c}\right)=(1.25,0.5)$ or $(X, Y)$ $=(50 \mathrm{~cm}, 20 \mathrm{~cm})$ [in the following, we use $\left(x_{c}, y_{c}\right)$ only to indicate the cylinder position; the corresponding dimensional coordinates $(X, Y)$ can be obtained by Eq. (6)], and the evolution was found to be roughly the same as in the symmetric case shown in Fig. 7, only with the central cell (around the cylindrical obstacle) slightly displaced.

The evolution is different, however, for the case of the obstacle positioned further off-center. This is illustrated in Fig. 8 for the configuration $\left(x_{c}, y_{c}\right)=(1.0,0.5)$. A striking difference with the symmetric case shown in Fig. 7 is the fact that the corner cells $L^{+}$and $R^{+}$that arise soon after the starting stage [Fig. 6(b)] have unequal sizes: $R^{+}$is a little larger than $L^{+}$[Fig. 8(a)]. As a result, the splitting of the original central cell $C^{-}$takes place in a different way, and the overall flow pattern soon loses its symmetry. The corner cells $L^{+}$and $R^{+}$again move towards the cylindrical obstacle, where they merge [Figs. 8(b) and 8(c)], although in a complicated way: At some stage three cyclonic satellite cells are observed around the cylinder [Fig. 8(c)]. Subsequently, the merged central cell is then seen to move away from the cylinder, and finally occupies a position close to the center of the container [Figs. 8(d) and 8(f)], where it remains. As in the symmetric case (Fig. 7), in the final stage the central cyclonic cell is accompanied by two anticyclonic cells thus forming a three-cell pattern, with the cylinder now in between the left-hand and central cells. The flow at this stage shows a stronger unsteadiness than in the symmetric case, in particular in the region around the cylinder: As a result of viscously generated vorticity at the cylinder wall, smaller eddies are formed continuously, which are subsequently advected by the larger cells, causing perturbations in particular to the left-hand cell.

The flow evolution changes dramatically when the cylindrical obstacle is positioned further off-center, as shown in Fig. 9 for the case $\left(x_{c}, y_{c}\right)=(0.75,0.5)$. As in the previous cases, two cyclonic corner cells arise soon after the initial starting stage, but now the $R^{+}$cell becomes considerably larger than the $L^{+}$cell, whose growth is hindered by the presence of the cylinder [Fig. 9(a)]. The original central cell $C^{-}$is squeezed into three parts: Two at the end walls of the tank and one in the center. The left-hand corner cell $L^{+}$ quickly moves towards the cylinder and soon surrounds it [Figs. 9(b) and 9(c)], upon which cell $R^{+}$moves towards the cylinder as well, where it merges with $L^{+}$[Figs. 9(d) and $9(\mathrm{e})]$. During this merging process, the central part of the original cell $C^{-}$is seen to disappear [Fig. 9(c)]. The quasisteady final stage is composed of mainly two cells: One cyclonic cell around the cylindrical obstacle, and the other anticyclonic. Some weaker flow structures are visible near the (a)

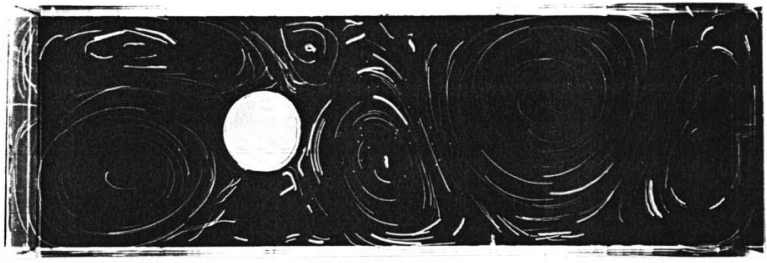

(b)

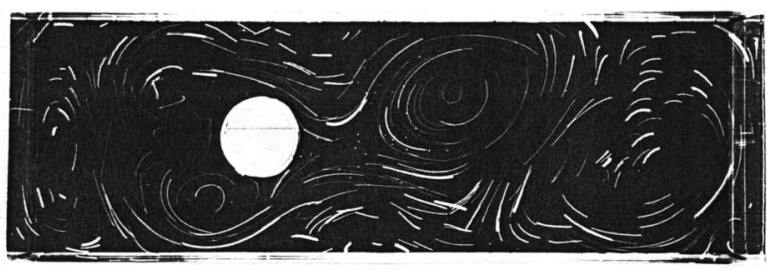

(c)

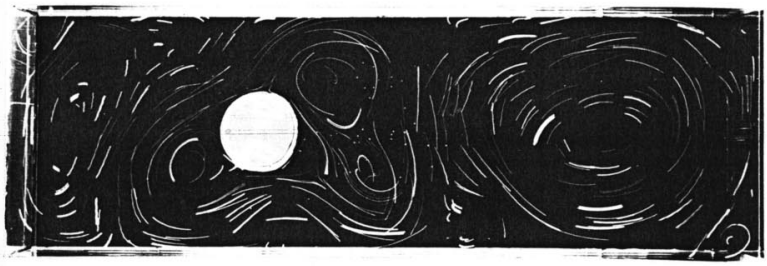

(d)

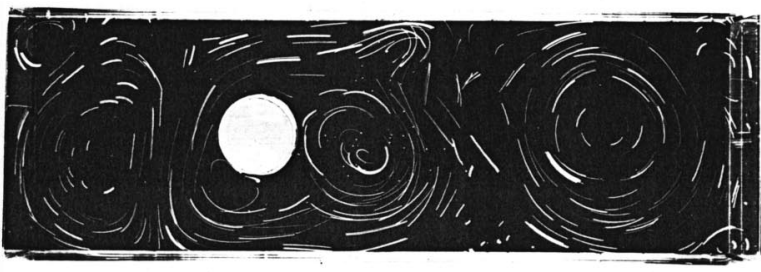

(e)

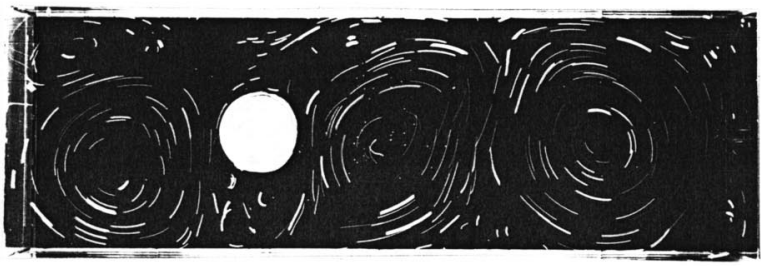

(f)

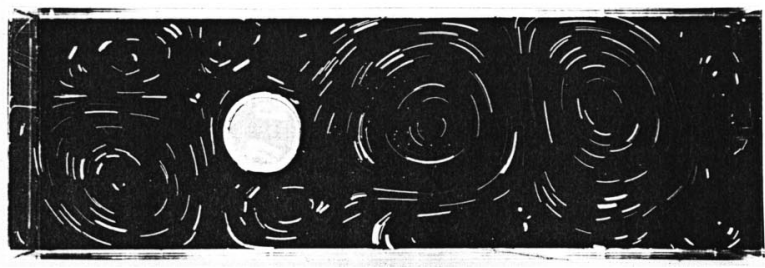

FIG. 8. Sequence of streak photographs illustrating the flow evolution in the case $\left(x_{c}, y_{c}\right)=(1.0,0.5)$ for $t=30 \mathrm{~s} \mathrm{(a),} 50 \mathrm{~s}$ (b), $1 \mathrm{~min}(\mathrm{c}), 1 \mathrm{~min} 20 \mathrm{~s}$ (d), $2 \mathrm{~min}(\mathrm{e}), 2 \mathrm{~min} 30 \mathrm{~s}(\mathrm{f})$. The exposure time is $2.5 \mathrm{~s}$.

end walls of the tank, but they are considerably less energetic than the main cells.

It is interesting to note that, although the obstacle positions in Figs. 8 and 9 differ only slightly, the final flow states are remarkably different. Apparently, when the cylinder is placed too close to the end wall (as in Fig. 9), the anticyclonic cell that is split off from the initial cell $C^{-}$is no longer able to grow and push the corner cell away towards the center of the tank (as observed in Fig. 8). In order to examine how critical the flow evolution depends on the obstacle position, laboratory experiments were performed for slightly different cylinder positions $\left(x_{c}, y_{c}\right)$ : While $y_{c}=0.5$ 
(a)

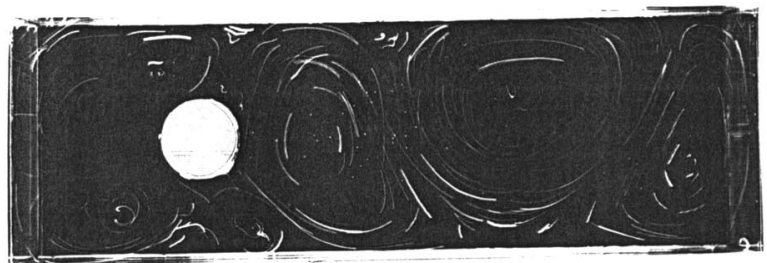

(b)

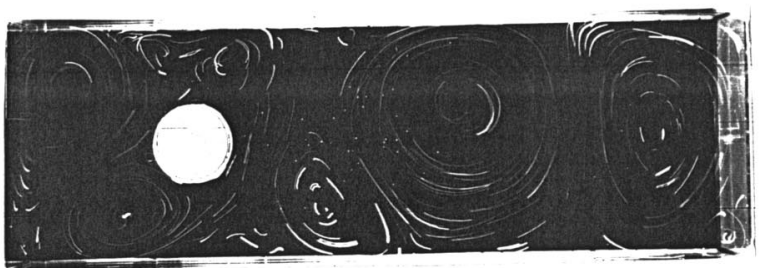

(c)

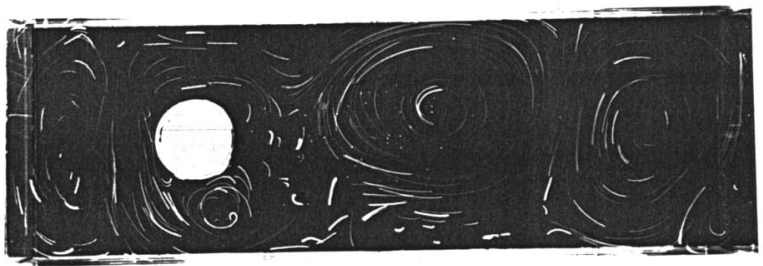

(d)

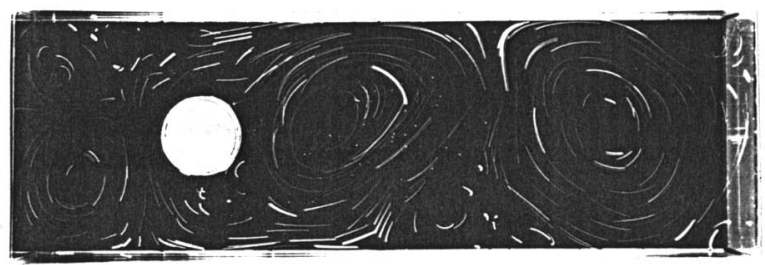

(e)

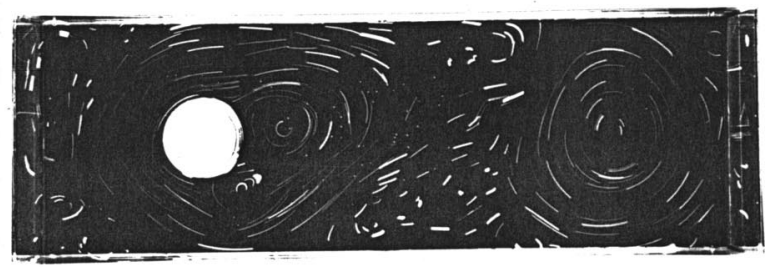

(f)

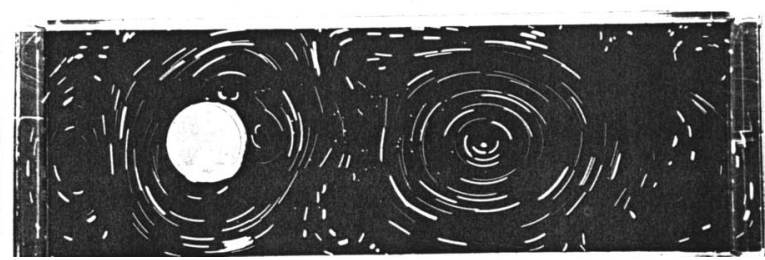

FIG. 9. Sequence of streak photographs illustrating the flow evolution in the case $\left(x_{c}, y_{c}\right)=(0.75,0.5)$ for $t=30 \mathrm{~s}(\mathrm{a}), 40 \mathrm{~s}(\mathrm{~b}), 50 \mathrm{~s}(\mathrm{c}), 1 \mathrm{~min} 10 \mathrm{~s}(\mathrm{~d}), 1$ $\min 50 \mathrm{~s}(\mathrm{e}), 5 \mathrm{~min}(\mathrm{f})$. The exposure time is $2.5 \mathrm{~s}$ for (a)-(e) and $4.5 \mathrm{~s}$ for (f).

was kept fixed, $x_{c}$ was changed around the value $x_{c}=1.0$. It was found that for $x_{c}=1.11$ and 1.08 the cylinder captures a cyclonic cell, as in the case $x_{c}=1.5$ shown in Fig. 7. For $x_{c}=0.93$ the cylinder does not capture any cyclonic cell, and the flow evolution is similar to that in the case $x_{c}=1.0$, shown in Fig. 8. For a smaller $x_{c}$-value $\left(x_{c}=0.83\right)$ we observed the capturing of the cyclonic corner cells and the subsequent formation of a two-cell pattern as in the case $x_{c}$ $=0.75$ shown in Fig. 9. Apparently, the final flow pattern is critically dependent on the cylinder's position.

In all the cases considered thusfar the cell $R^{+}$was observed to move from its origin (the upper right corner of the (a)

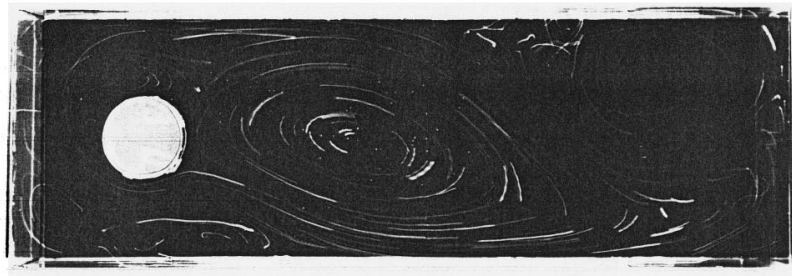

(b)

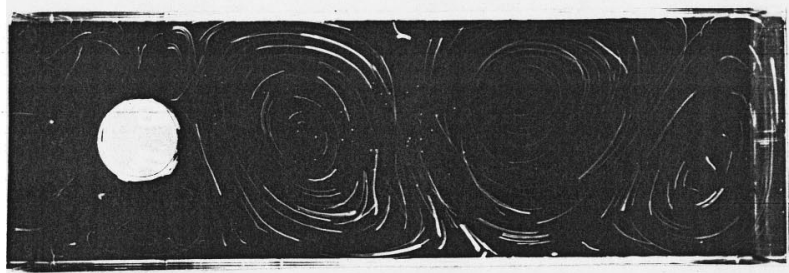

(c)

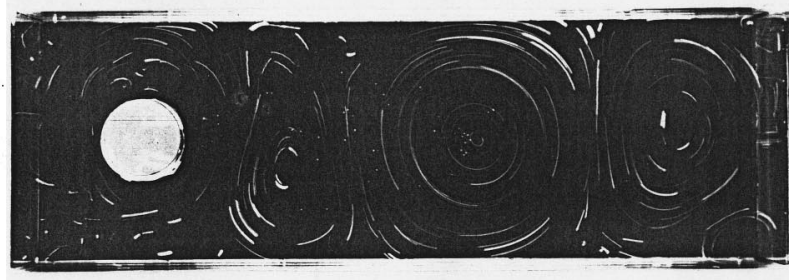

(d)

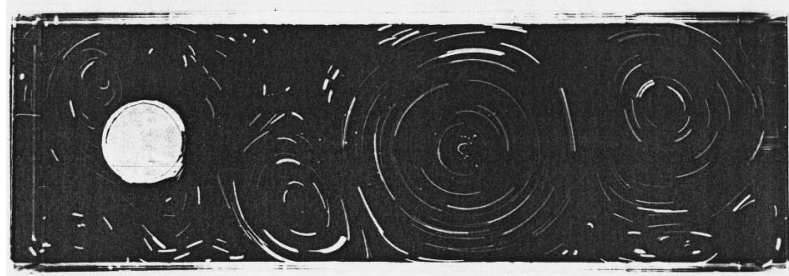

(e)

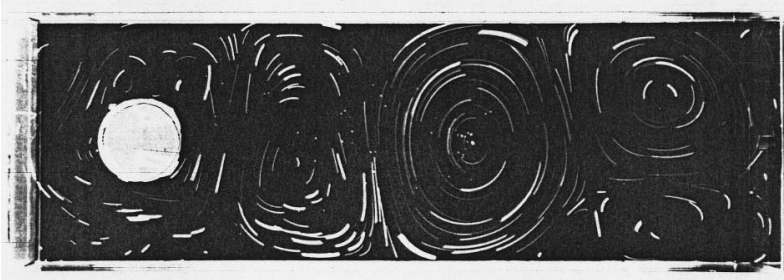

(f)

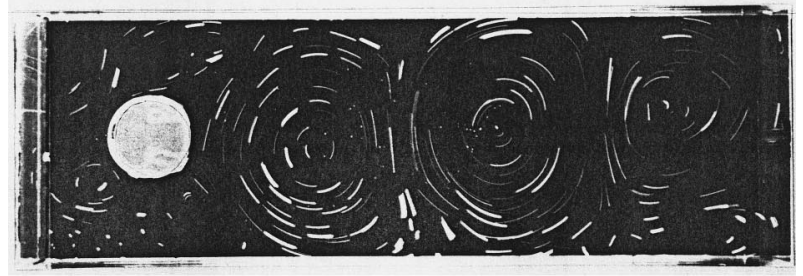

FIG. 10. Sequence of streak photographs illustrating the flow evolution in the case $\left(x_{c}, y_{c}\right)=(0.5,0.5)$ for $t=20 \mathrm{~s}(\mathrm{a}), 30 \mathrm{~s}(\mathrm{~b}), 1 \mathrm{~min}(\mathrm{c}), 1 \mathrm{~min} 50 \mathrm{~s}$ (d), $3 \mathrm{~min}(\mathrm{e}), 4 \mathrm{~min}$ (f). The exposure time is $2.5 \mathrm{~s}$ for (a) $-(\mathrm{d})$ and $3.5 \mathrm{~s}$ for (e) and (f)

rectangular domain) towards the cylindrical obstacle, where it finally merged with the cell $L^{+}$. When the cylinder is located even further away from the center, however, the cell $R^{+}$is no longer able to reach the cylinder, and merging with $L^{+}$does not take place. The flow evolution then follows a different scenario, as illustrated in Fig. 10 for the case $\left(x_{c}, y_{c}\right)=(0.5,0.5)$. Initially, $R^{+}$is seen to drift towards the center [Figs. 10(a)-10(d)] while $C^{-}$shrinks as in the previous cases. At some stage [see Fig. 10(d)] the position of $R^{+}$ becomes more or less stationary, and $C^{-}$re-establishes itself 
(a)

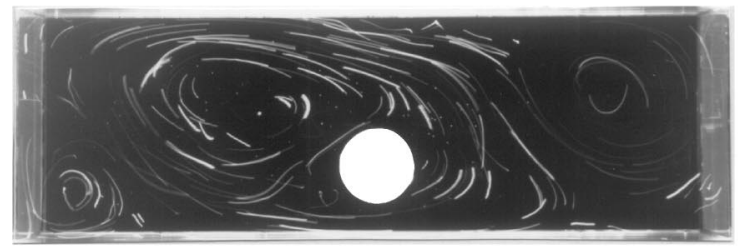

(b)

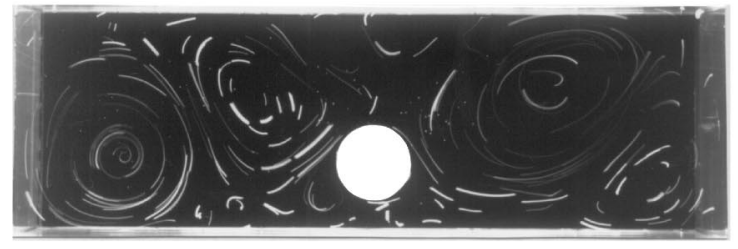

(c)

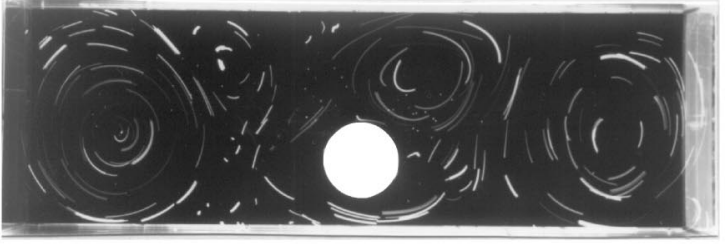

(d)

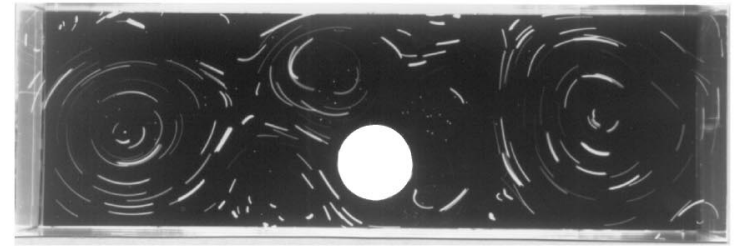

(e)

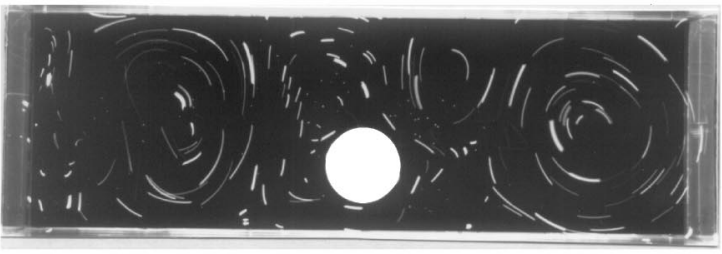

(f)

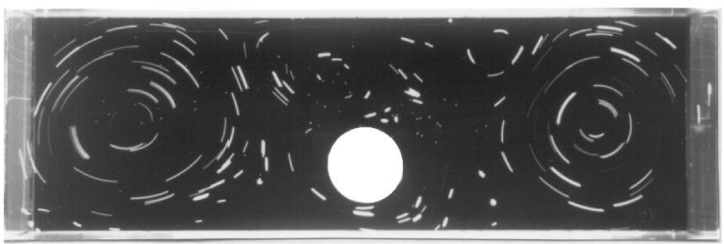

FIG. 11. Sequence of streak photographs illustrating the flow evolution in the case $\left(x_{c}, y_{c}\right)=(1.5,0.33)$ for $t=30 \mathrm{~s}(\mathrm{a}), 50 \mathrm{~s}(\mathrm{~b}), 1 \mathrm{~min} 10 \mathrm{~s}(\mathrm{c}), 1 \mathrm{~min}$ $30 \mathrm{~s}(\mathrm{~d}), 1 \mathrm{~min} 50 \mathrm{~s}(\mathrm{e}), 4 \mathrm{~min}(\mathrm{f})$. The exposure time is $2.5 \mathrm{~s}$ for (a)-(e) and $3.5 \mathrm{~s}$ for (f).

[Fig. 10(e)]. The final state [Fig. 10(f)] is characterized as three primary cells (the central cell being cyclonic) plus one weaker cyclonic cell around the cylindrical obstacle.

Laboratory experiments were also performed with cylinder positions off the container's long axis $\mathrm{CH}$ (i.e., $y_{c}$ $\neq 0.5)$. Figure 11 shows the flow evolution for the case $\left(x_{c}, y_{c}\right)=(1.5,0.33)$. After the starting stage, the cyclonic corner cells arise as before, but the cell $R^{+}$grows much more rapidly than its counterpart $L^{+}$[Fig. 11(a)]. Cell $R^{+}$ moves towards the cylinder [Figs. 11(b) and 11(c)], passes it and merges with cell $L^{+}$[Fig. 11(d)]. In the meantime an intense anticyclonic cell has formed at the right-hand end wall off the container [Figs. 1(a) and 1(b)], which is persistent and remains there during the further evolution. In the final stage the flow has become organized into two main cells (a cyclonic cell on the left-hand side, and an anticyclonic one on the other side), while some irregular weaker structures are observed in the vicinity of the obstacle.

Additional experiments were carried out for $y_{c}=0.33$ while the $x_{c}$-position of the cylinder was varied: $x_{c}=0.5$, 1.0, 2.0, and 2.5. In neither of these cases we observed the formation of a cell around the cylindrical obstacle during the quasi-steady final stage of the evolution. This noncapturing of a main cell is presumably owing to the tendency of the main cells to occupy a position on the longer axis $\mathrm{CH}$ of the container, which is incompatible with the off-axis position of the cylinder.

\section{DISCUSSION}

The laboratory experiments have clearly revealed that the presence of an obstacle in the container plays a crucial role in the spin-up process. Firstly, because an obstacle acts as a barrier in the flow, its impermeability locally imposing the condition of zero normal velocity. Secondly, because the obstacle acts as a source of viscously produced vorticity, the no-slip condition implies the presence of a boundary layer. This latter property is of importance to the flow evolution, both in the initial stage and in subsequent, later stages. An example can be found in the starting flow, as visualized for a number of different obstacle positions in Fig. 6. The barrier effect is clearly visible from these streak photographs (compare with the starting flow pattern in absence of any obstacle, see vHDD); the shape of the starting flow pattern is reasonably well predicted by the inviscid "potential flow" solution presented in Sec. III. As discussed in the beginning of Sec. IV, however, some discrepancies can be observed for the cases in which the obstacle is placed off the long axis of the container, see Figs. 6(d)-6(f): On the left-hand side of the obstacle (i.e., downstream, with respect to the overall anticyclonic motion of the fluid) the cells in the laboratory experiment are larger than theoretically predicted. This feature is attributed to "feeding" by negative vorticity originating from the boundary layer at the obstacle, which is stripped off by the primary flow.

In order to appreciate the role of the obstacle as a source of vorticity in the starting stage of the adjustment process, it is useful to compare the vorticity distributions in the cases with and without obstacle. In the absence of any internal obstacle, the starting flow is characterized by a relative flow with uniform vorticity $\omega_{0}=-2 \Delta \Omega$ (with $\Delta \Omega$ the increase in rotation speed) all over the domain, while a boundary layer is present at the lateral boundaries; the vorticity in this boundary layer is positive. The no-slip condition at the lateral boundaries implies that the total circulation of the relative flow is zero

$$
\oint_{c} \mathbf{v} \cdot \mathbf{d r}=\iint_{A} \omega_{z} d A=0
$$

with $\mathbf{d r}$ an element of the contour $C$ (which corresponds with the lateral boundary) and $A$ the area of the domain. Appar- 
ently, the total (i.e., area-integrated) amount of positive vorticity present in the boundary layer is $2 \Delta \Omega B L$, in order to satisfy (16).

Let us now consider the configuration with the internal obstacle. The starting flow is again characterized by a relative flow with uniform vorticity $\omega_{0}=-2 \Delta \Omega$ (for which an analytical description is given in Sec. III) all over the domain, with a boundary layer containing positive vorticity at the lateral boundaries, but now with an additional boundary layer at the obstacle's surface. In contrast to the boundary layer at the container walls, however, the obstacle's boundary layer contains a net amount of negative vorticity, because the primary-flow direction around the obstacle is counterclockwise (Fig. 3); the perimeter of the circle that is in contact with the eddies has positive vorticity but it is in overall weaker than that the primary flow has. In order to clarify this point, we assume for convenvience that the boundary layers are still extremely thin, and that the distance between the obstacle and the nearest lateral boundary is large compared to the boundary layer thickness. In that case, it is assumed that the lateral boundary layer has approximately the same structure as in the case without any internal obstacle, and thus contains a total (area-integrated) amount of positive vorticity $2 \Delta \Omega B L$. Due to the presence of the cylindrical obstacle, the area-integrated vorticity in the interior flowoutside the boundary layers-measures $-2 \Omega \times$ area $=-2 \Omega\left(B L-\pi a^{2}\right)$, with $a$ the cylinder radius. In order to satisfy the condition (16) of zero net vorticity, the boundary layer at the obstacle has to carry a total vorticity $-2 \Omega \pi a^{2}$.

The sign of this vorticity is in contrast with that in the boundary layer at the outer wall, which contains positive vorticity. In order to appreciate the subtle differences between inner and outer boundaries, we may change the topology of the flow by adding a barrier that connects the obstacle with the outer boundary: The flow configuration then changes from a doubly connected to a singly connected domain. In the latter case, the obstacle forms a part of the outer boundary (and so do either sides of the barrier), and hence will carry a boundary layer containing positive vorticity. In that case, the initial flow pattern and the subsequent evolution will be completely different from those in the doubly connected configuration.

Until now we have mainly considered the initial stage of the spin-up process, i.e., the starting flow. Separation of the boundary layers will occur very rapidly, leading to filamentary structures that roll up into larger vortex structures. For the boundary layer at the container walls this leads to the formation of the corner cells $\left(R^{+}\right.$and $\left.L^{+}\right)$, as visible, e.g., in Fig. 7(a). For the case that the obstacle is placed off the long axis of the container, the flow separation at the obstacle (i.e., the stripping of its negative-vorticity boundary layer) results in the feeding of the cell at its downstream side, as can be seen in the streak photographs Figs. 6(d)-6(f). At later stages, the larger cellular structures in the flow show a complicated interaction with the obstacle. As a rule, a neighboring cell with positive (negative) circulation gives rise locally to a boundary layer with negative (positive) vorticity. It may be expected that these boundary layers are stripped off the obstacle, leading to thin filamentary vorticity structures that are advected into the interior of the flow domain. In this sense, the obstacle acts as a continuous source of small-scale vorticity. Of course, these features cannot be observed from the flow visualizations shown in Figs. 7-11, but their occurrence is anticipated from high-resolution spectral simulations of 2D turbulent flows near rigid no-slip walls. ${ }^{22}$

During the course of the experiments it was noticed that whenever the cylinder was enclosed by a vortex, in the majority of cases it was a cyclonic one, while only in a very few cases it was a comparitively weak anticyclonic vortex. For instance in Fig. 10, the cylinder is at first surrounded by a primary anticyclonic cell [Fig. 10(a)]. This cell is soon detached [Fig. 10(b)] and instead a cyclonic cell encompasses the cylinder [Fig. 10(c)]. In fact in this case the cylinder is located exactly where an anticyclonic cell would be present if the spin-up were taking place in the absence of the cylindrical obstacle (this also applies more or less to the experiment shown in Fig. 9). This suggests that there exists some difference in stability characteristics between the cyclonic and anticyclonic vortices around the cylinder subjected to a background rotation.

Kloosterziel and van Heijst ${ }^{23}$ extended Rayleigh's centrifugal instability criterion to explain why it is extremely difficult to create a single anticyclonic barotropic vortex in the laboratory. The extended criterion for stability of an axisymmetric flow with azimuthal velocity $v_{\theta}(r)$ in a frame that rotates with angular speed $\Omega$ reads

$$
\frac{d}{d r}\left(r v_{\theta}+\Omega r^{2}\right)^{2} \geqslant 0,
$$

or, in slightly different notation

$$
\left(v_{\theta}+\Omega r\right)\left(\omega_{z}+2 \Omega\right) \geqslant 0,
$$

with $\omega_{z}=[(1 / r)(d / d r)]\left(r v_{\theta}\right)$. From this criterion, it can be easily verified that a swirling flow encircling the cylindrical obstacle in cyclonic sense (with $v_{\theta}>0$ and $\omega_{z}>0$ ) is generally stable for moderate Rossby numbers, while an anticyclonic swirling motion (with $v_{\theta}<0$ and $\omega_{z}<0$ ) is not.

\section{ACKNOWLEDGMENT}

The financial support of the Korea Science and Engineering Foundation (Grant No. 95-0200-06-01-3) to Y.K.S. is gratefully acknowledged.

${ }^{1}$ H. P. Greenspan and L. N. Howard, "'On a time-dependent motion in a rotating fluid,' J. Fluid Mech. 17, 385 (1963).

${ }^{2}$ G. Venezian, “Nonlinear spin-up,' Topics Ocean Eng. 2, 87 (1970).

${ }^{3}$ P. D. Weidman, "On the spin-up and spin-down of a rotating fluid,' J. Fluid Mech. 77, 685 (1976).

${ }^{4}$ W. B. Watkins and R. G. Hussey, "Spin-up from rest in a cylinder,' Phys. Fluids 20, 1596 (1977).

${ }^{5}$ E. R. Benton, "Vorticity dynamics in spin-up from rest,', Phys. Fluids 22, 1250 (1979).

${ }^{6}$ J. M. Hyun, F. Leslie, W. W. Fowler, and A. Warn-Varnas, "Numerical solutions for spin-up from rest in a cylinder,' J. Fluid Mech. 127, 263 (1983).

${ }^{7}$ J. A. van den Konijnenberg and G. J. F. van Heijst, " Non-linear spin-up in a circular cylinder,', Phys. Fluids 7, 2989 (1995).

${ }^{8}$ G. J. F. van Heijst, "Spin-up phenomena in non-axisymmetric containers,' J. Fluid Mech. 206, 171 (1989).

${ }^{9}$ G. J. F. van Heijst, P. A. Davies, and R. G. Davis, “Spin-up in a rectangular container,'’ Phys. Fluids A 2, 150 (1990). 
${ }^{10}$ H. I. Andersson, J. T. Billdal, and G. J. F. van Heijst, "Spin-up in a semicircular container," Int. J. Numer. Methods Fluids 15, 503 (1992).

${ }^{11}$ J. A. van den Konijnenberg, H. I. Andersson, J. T. Billdal, and G. J. F. van Heijst, "Spin-up in a rectangular tank with low angular velocity," Phys. Fluids 6, 1168 (1994).

${ }^{12}$ Y. K. Suh, "Numerical study on two-dimensional spin-up in a rectangle," Phys. Fluids 6, 2333 (1994).

${ }^{13}$ J. A. van den Konijnenberg, T. L. Wessels, and G. J. F. van Heijst, "Spin-up in a circular tank with a radial barrier," Phys. Fluids 8, 2048 (1996).

${ }^{14}$ J. A. van den Konijnenberg and G. J. F. van Heijst, "Free-surface effects on spin-up in a rectangular tank," J. Fluid Mech. 334, 189 (1997).

${ }^{15}$ D. M. Henderson, J. M. Lopez, and D. L. Stewart, "Vortex evolutions in non-axisymmetric impulsive spin-up from rest," J. Fluid Mech. 324, 109 (1996).

${ }^{16}$ G. J. F. van Heijst and H. J. H. Clercx, "Self-organisation of quasi-2D flows in a rectangular container," Appl. Sci. Res. 58, 149 (1998).
${ }^{17}$ G. J. F. van Heijst, L. R. M. Maas, and C. W. M. Williams, “The spin-up of fluid in a rectangular container with a sloping bottom,' J. Fluid Mech. 265, 125 (1994)

${ }^{18}$ J. A. van den Konijnenberg and G. J. F. van Heijst, "Spin-up in a rectangular tank with discontinuous topography,' Phys. Fluids 8, 2943 (1996).

${ }^{19}$ J. A. van den Konijnenberg, H. I. Andersson, and G. J. F. van Heijst, "Spin-up in a rectangular tank with a sloping bottom," Eur. J. Mech. B/Fluids 16, 609 (1997).

${ }^{20}$ J. Pedlosky, Geophysical Fluid Dynamics (Springer-Verlag, Berlin, 1982).

${ }^{21}$ L. M. Milne-Thomson, Theoretical Hydrodynamics (Macmillan, London, 1968).

${ }^{22}$ H. J. H. Clercx, S. R. Maassen, and G. J. F. van Heijst, "Spontaneous spin-up during the decay of $2 \mathrm{D}$ turbulence in a square container with rigid boundaries," Phys. Rev. Lett. 80, 5129 (1998).

${ }^{23}$ R. C. Kloosterziel and G. J. F. van Heijst, "An experimental study of unstable barotropic vortices in a rotating fluid," J. Fluid Mech. 223, 1 (1991). 